\title{
Determination of soil grain size composition by measuring apparent weight of float submerged in suspension
}

\author{
Jarosław Kaszubkiewicz ${ }^{1 *}$, Witold Wilczewski², Tibor József Novák³, Przemysław Woźniczka ${ }^{1}$ \\ Krzysztof Faliński², Jerzy Belowski², and Dorota Kawatko ${ }^{1}$ \\ ${ }^{1}$ Institute of Soil Science and Environment Protection, Wrocław University of Environmental and Life Sciences, \\ Grunwaldzka 53, 50-357 Wrocław, Poland \\ ${ }^{2}$ Arcanum Sp. z o.o., Parkowa 6/1, 51-616 Wrocław, Poland \\ ${ }^{3}$ Department of Landscape Protection and Environmental Geography, University of Debrecen, \\ Böszörményi út 138,4032, Debrecen, Hungary
}

Received March 23, 2016; accepted December 13, 2016

\begin{abstract}
A b s t r a c t. Texture is one of the most significant physical properties of soils. Over the years, several methods of its measuring were developed. The paper presents a method for determining the particle size composition of soils, based on the separation of particles in the sedimentation process. Density of suspension is determined on the basis of apparent weight changes of a float submerged in it. The weight of the float suspended on a thin line, at a given depth in the suspension, is measured with a sensitive piezoelectric dynamometer. The Stokes equation is used to calculate the content of soil fractions with equivalent diameters in the range of 0.001 to $0.1 \mathrm{~mm}$. Digital transmission of results from the dynamometer, the temperature sensor and measurements of the distance defining the depth of immersion of the float to the computer enable calculations of particle size composition to be performed automatically. This paper presents the results of measurements of the particle size composition of artificially generated mixtures of 'silt' and 'clay'. The results are compared with results obtained with other methods (including the laser method). A high level of repeatability of the results and satisfactory compatibility in relation to the reference pipette method are noted.

K e y w o r d s: sedimentation, particle size composition, soil suspension density
\end{abstract}

\section{INTRODUCTION}

Particle size composition is the primary parameter necessary for the evaluation of other soil properties and for the determination of soil agricultural suitability (Sławiński et al., 2011; Smith, Mullins, 1991). A significant group of methods for its determination are sedimentation methods developed over the past 100 years (Allen et al., 1996; Allen, 1997, Ryżak et al., 2009). Their primary objective is

*Corresponding author e-mail: jaroslaw.kaszubkiewicz@up.wroc.pl to determine the share of the mass of selected fractions of soil. These methods have been improved over many years (Bieganowski and Walczak, 2004; Gee and Bauder, 1979; Rząsa, 1978), and currently, together with the laser diffraction methods developed in recent decades (Agrawal et al., 1991; Bieganowski and Walczak, 2004; Bieganowski et al., 2010; Singer et al., 1988), are a basic research tool (ISO 11277, 1998; Rząsa and Owczarzak, 2013). The pipette method (SPM) is accepted in many countries as the official reference method for measuring the fraction of equivalent diameters less than $63 \mu \mathrm{m}$ (Allen, 1997; ISO, 1998). It is considered an accurate method but time-consuming and laborious (Allen, 1997; Beusenlick et al., 1998; Ryżak et al., 2009), which limits its use in mass measurements.

The cited laser diffraction methods (LDM) are characterized by many advantages, among which the most important are the speed and repeatability of the analysis and the wide range of the designated fraction. However, as shown in reports by many authors (Agraval et al., 1991; Allen, 1997; Di Stefano et al., 2010; Konert and Vandenberghe, 1997; Ryżak and Bieganowski, 2010), their different physical principles imply different results from those obtained in sedimentation methods.

All sedimentation methods are based on the assumption that soil particles in water suspension behave according to the Stokes equation which was applied to soil analysis by Oden (2010). According to the equation, fall time for particle with diameter to depth is proportional to the square of the diameter. This law is satisfied by spherical particles that

(C) 2017 Institute of Agrophysics, Polish Academy of Sciences 
comply with additional conditions, which will be discussed later in this paper. The speed of descent of an actual grain may substantially differ from that calculated for spherical particles (Dietrich, 1982; Loth, 2008). The problem of the influence of particle shape on results obtained for all methods including laser methods should be noted (Eshel et al., 2004; Sochan et al., 2012). LDM methods seem to be independent of the heterogeneity of the density of soil particles of various sizes, which has an impact on sedimentation (Bah et al., 2009).

In sedimentation methods the content of solid phase particles in suspension is measured, where as a result of the different speeds of descent of grains the separation of soil particles of different sizes occurs. The measurement is carried out via the evaporation of water (PM) or by measuring the density of the suspension. Measuring the density of the suspension can be realized using a Casagrande or Prószyński aerometer (SHM) (Komornicki and Jakubiec, 1978; Kovács et al., 2006) or by measurement of hydrostatic pressure that acts in the suspension (Kovács et al., 2004; Nemes et al., 2002). Measuring of light absorption or other types of radiation (Oliveira et al., 1997; Ryżak et al., 2009) is also used for this purpose.

An interesting variation of the sedimentation methods is the determination of weight of sedimentation particles settling on a pan immersed in suspension as a function of time (Gliński and Konstankiewicz, 1991). This is a time-consuming method; however, it is similar to the pipette method and furthermore it provides the possibility of simultaneous determination of multiple samples in a single device.

It seems, therefore, that it would be advantageous to introduce such a modification to the sedimentation methods, while avoiding, at least in part, their shortcomings. This new method would at the same time give similar results with reduced time outlay.

We propose a method which should combine the accuracy of results obtained using the pipette method with the possibility of measuring any number of selected size fractions and a shortened measurement time in comparison to the SHM and PM methods.

To describe the theoretical basis of the proposed measurement method it is necessary to recognise physical forces affecting the float immersed in water suspension of soil particles with different equivalent diameters. The float has a higher density than the soil suspension and it is hanging on a thin line connected to a high resolution dynamometer. The net force $(G)$ acting on the float immersed to depth $z$ in soil suspension after time $t$ when the sedimentation starts is equal to:

$$
G(z, t)=\left(M_{f}-V_{f} \rho(z, t)\right) g,
$$

where: $G(z, t)$ - net force acting on the float submerged in the soil suspension at a depth $z$ after time $t$ (apparent weight of the float measured with a dynamometer);
$M_{f}$ - mass of float; $V_{f}$ - volume of float; $\rho(z, t)$ - density of soil suspension at the depth $z$ after the time $t$; $g$ - gravitational acceleration.

The above equation is approximate due to changes in the density of the suspension at the height of the float. Soil suspension density at depth $z$ after sedimentation time $t$ can be calculated by dividing the total weight of the solid phase and the water in the layer with thickness $d z$ by the volume of that layer:

$$
\begin{aligned}
& \rho(z, t)=\frac{d M_{w}(z, t)+d M_{s}(z, t)}{s d z}= \\
& \frac{\rho_{w} d V_{w}(z, t)+d M_{s}(z, t)}{s d z}= \\
& \frac{\rho_{w}\left(s d z-\frac{d M_{s}(z, t)}{\rho_{s}}\right)+d M_{s}(z, t)}{s d z},
\end{aligned}
$$

where: $d M_{s}(z, t)$ - mass of the solid phase of soil suspension at the layer having a thickness $d z$ at a depth $z$ after time $t ; d M_{w}(z, t)$ - mass of water at the suspension layer having a thickness $d z$ at a depth $z$ after time $t ; d V_{w}(z$, $t$ ) - volume of water at the suspension layer having a thickness $d z$ at a depth $z$ after time $t ; \rho_{w}$ - density of water; $\rho_{s}$-density of solid phase of soil.

It is assumed in Eq. (2) that the density of particles of varying sizes is constant, or may by slightly different, and it is possible to approximate this with the single value $\rho_{s}$

The mass of soil solid phase in the suspension layer with thickness $d z$ at depth $z$ at time $t$ can be calculated from the equation:

$$
d M_{s}(z, t)=s d z \frac{M}{V_{z}} F(z, t),
$$

where: $M$ - total mass of the solid phase of soil suspension (total mass of soil particles); $V_{z}$ - total volume of suspension; $F(z, t)$ - proportion soil of particles which are in a unit volume of suspension at a depth $z$ after time $t$, related to the total weight of soil the particles.

So, the density of suspension at depth $z$ after time $t$ can be described as:

$$
\begin{aligned}
& \rho(z, t)=\frac{\rho_{w}\left(s d z-s d z \frac{M}{V_{z}} \frac{F(z, t)}{\rho_{s}}\right)+s d z \frac{M}{V_{z}} F(z, t)}{s d z}= \\
& \rho_{w}\left(1-\frac{M}{V_{z}} \frac{F(z, t)}{\rho_{s}}\right)+\frac{M}{V_{z}} F(z, t)= \\
& =\rho_{w}+\frac{M}{V_{z}} F(z, t)\left(1-\frac{\rho_{w}}{\rho_{s}}\right) .
\end{aligned}
$$

After modification of Eq. (4) to calculate function $F(z, t)$ we obtain: 


$$
F(z, t)=\frac{V_{z}}{M} \frac{\rho_{s}\left(\rho(z, t)-\rho_{w}\right)}{\left(\rho_{s}-\rho_{w}\right)} .
$$

Transforming Eq. (1) to calculate the density of soil suspension we obtain:

$$
\rho(z, t)=\frac{\left(M_{f} g-G(z, t)\right.}{V_{f} g},
$$

where: $g$ - gravitational acceleration.

Substituting Eq. (6) to Eq. (5) we obtain:

$$
\begin{aligned}
& F(z, t)=\frac{V_{z}}{M} \frac{\rho_{s}\left(\rho(z, t)-\rho_{w}\right)}{\left(\rho_{s}-\rho_{w}\right)}= \\
& \frac{V_{z}}{M} \frac{\rho_{s}}{\left(\rho_{s}-\rho_{w}\right)}\left(\frac{\left(M_{f} g-G(z, t)\right)}{V_{f} g}-\rho_{w}\right) .
\end{aligned}
$$

The above Eq. (7) defines the relationship between the apparent weight of the float, submerged to depth $z$ below the suspension surface, at time $t$ from the beginning of sedimentation (mixing) and the cumulative fraction content of particles that fall to depth $z$ by time $t$.

Considering the fact that the density of water is dependent on temperature, the above equation should in principle be written as:

$$
F(z, t)=\frac{V_{z}}{M} \frac{\rho_{s}}{\left(\rho_{s}-\rho_{w}\right)}\left(\frac{\left(M_{f} g-G(z, t)\right)}{V_{f} g}-\rho_{w}(\tau)\right),
$$

where: $\tau$ - temperature of suspension.

The function $\rho_{w}(\tau)$ in the temperature range $18-25^{\circ} \mathrm{C}$ (such as occurs most frequently in sedimentation measurements) can be approximated with sufficient accuracy using a polynomial of the second order.

Settling velocity of a spherical particle of diameter $d$ and density $\rho_{s}$ in a fluid with density $\rho_{w}$ and dynamic viscosity $\eta$ can be calculated from the equilibrium condition of the forces acting on it:

$$
v=\frac{d^{2} g}{18 \eta}\left(\rho_{s}-\rho_{w}\right),
$$

where: $v$ - settling velocity of a spherical particle of diameter d.

The above equation can be applied to spherical particles, in the range of the laminar flow (Reynolds number $<1$ ), and to the diameter of the container (cylindrical) wherein the sedimentation occurs that is much greater than the diameter of falling particles and there is an absence of interactions between particles (Allen, 1997; Smith and Mullins, 1991).

At the same time, the diameter of the falling particles must be greater than the limit at which the thermal motions keep the particles in suspension. According to Allen et al. (1996), this particle diameter limit, at which Brownian motion may move soil particles larger than the distance of movement caused at the same time by the force of gravity, is about $1 \mu \mathrm{m}$.

The distance which particles are moved during sedimentation with velocity $v$ at time $t$ will be:

$$
z=v t=\frac{d^{2} g}{18 \eta}\left(\rho_{s}-\rho_{w}\right) t
$$

where: $d$ - diameter of soil particle, $\eta$ - dynamic viscosity of water.

With the little variability of the density of soil particles belonging to the different size fractions and in conditions of constant temperature the following substitution can be assumed:

$$
\alpha=\text { const }=\frac{g}{18 \eta}\left(\rho_{s}-\rho_{w}\right) .
$$

In fact, $\alpha$ is a function of temperature, because both $\rho_{w}$ and $\eta$ depend on temperature. Based on the above substitution, Eq. (10) can be, therefore, written as:

$$
z=\alpha d^{2} t
$$

Equation (12) defines the depth to which, after time $t$, a particle with equivalent diameter $d$ and located close to the surface of the suspension at the initial time of sedimentation has fallen.

Using a dynamometer to measure the force acting on the float submerged in the soil suspension $G(z, t)$ (the apparent weight of the float) at desired depth $z$ with the appropriately selected times $t_{1}, t_{2}, \ldots t_{n}$, it is possible, on the basis of Eq. (8) to determine the values of the function $F(z, t)$ for that times.

Let us choose the depth at which the measurements of float apparent weight will be made and define this as $z_{p}$. Selections can be made precisely because the dynamometer works without a spring being exposed during the measurement (the piezoelectric effect is used).

Fall time of soil particles with diameter $d_{1}$ from the surface of the suspension to depth $z_{p}$ can be calculated after transformation of Eq. (12):

$$
t_{1}=\frac{z_{p}}{\alpha d_{1}^{2}} .
$$

Of course, all particles of size greater than $z_{p}$ and those located, at the initial time of sedimentation, at any depth below the suspension surface, fall quicker than calculated above. According to the selected diameters $d_{2}, d_{3}, \ldots d_{n}$ the sedimentation times $t_{2}, t_{3}, \ldots t_{n}$ are calculated using Eq. (13).

It is important that the measuring depth $z_{p}$ is fixed and can be freely selected by the experimenter by raising or lowering the dynamometer with the float hooked to it on a thread, but not, as is the case of the hydrometer method, defined by an unknown content of soil particles with a diameter lower than that measured in real time. 
Factor $\alpha$ in Eq. (12) depends on temperature, and therefore must be written as:

$$
\alpha(\tau)=\frac{g}{18 \eta(\tau)}\left(\rho_{s}-\rho_{w}(\tau)\right) .
$$

The dynamic viscosity of water $\eta(\tau)$ in the temperature range of $18-25^{\circ} \mathrm{C}$ (such as occurs most frequently in measurements using the hydrometer method) can be approximated as a function of temperature, similar to the water density function, with the same acceptable accuracy. To achieve this, a polynomial of the second order may be used.

Substituting Eq. (14) into Eq. (13), we obtain time values for fall particles with required equivalent diameters. Time is calculated according to measurements of depth and changes of temperature. Temperature correction is an important value and it should not be overlooked.

Using a thin thread $(\phi \leq 0.1 \mathrm{~mm})$, on which the float is suspended, we avoid the errors which, in the hydrometric method, are associated with the displacement of the hydrometer stem submerging at a variable depth, depending on the content of the measured fraction.

For the times $t_{1}, t_{2}, \ldots t_{n}$ calculated in the above manner we measure, using a dynamometer, the values of the function $G\left(z_{p}, t_{i}\right)$, (apparent weight of the float submerged in suspension at the depth $z_{p}$ ), and then, using Eq. (8), we calculate the values of the expression $F\left(z_{p}, t_{i}\right)$. Content of fractions with diameters from $d_{i}$ to $d_{i+1}$ should be then calculated from the equation:

$$
f\left(d_{i}, d_{i+1}\right)=F\left(z_{p}, t_{i}\right)-F\left(z_{p}, t_{i+1}\right) .
$$

where: $f\left(d_{i}, d_{i+1}\right)$ - fraction of soil particles with the diameters from $d_{i}$ to $d_{i+1}$.

The values $d_{i}$ and $t_{i}$ are related by Eq. (13).

The choice of the depth at which the measurement is performed is determined by practical reasons. If we want to measure the fraction falling rapidly, it is convenient to choose a greater depth $z_{p}$ to make it possible to lower the float to the desired depth before the first measurement time (fraction with the largest diameter) and reduce the fluid turbulence, which is a result of the suspension mixing before the start of sedimentation. On the other hand, if we want to measure the content of small particles, it is convenient to perform measurements at a shallow depth $z_{p}$ which will give us a significant reduction (up to $3 \mathrm{~h}$ ) of measurement time for the content of a fraction with diameters less than $0.002 \mathrm{~mm}$.

Due to the non-zero volume of the float, the method of sedimentation $z_{p}$ should be set for the suspension with the immersed float. Hence, the suspension height of the entire cylinder $\left(H_{1}\right)$ with the submerged float is:

$$
H_{1}=\frac{V_{z}+V_{f}}{s}=\frac{V_{z}}{s}+\frac{V_{f}}{s}=H_{0}+\frac{V_{f}}{s},
$$

where: $H_{0}$ is the height of the suspension in a cylinder without a submerged float, $S$ - cross section area of cylinder.

So, if we want to place the centre of buoyancy of the float at depth $z_{p}$ below the surface, it should be placed above the bottom of the cylinder at the level:

$$
L_{1}=H_{0}+\frac{V_{f}}{s}-z_{p},
$$

where: $L_{1}$ - the distance from the bottom to the centre of buoyancy of the float.

The above equation indicates that, for a float with volume of approximately $40 \mathrm{~cm}^{3}$ in a standard cylinder with a diameter of $61 \mathrm{~mm}$, the correction is about $14 \mathrm{~mm}$.

Measurement of suspension density in this method was performed by measuring the apparent weight of the float attached to a sensitive dynamometer and submerged in the suspension. The float was made of glass and had the shape of two cones joined by their bases. The diameter of the base of the cone was $4 \mathrm{~cm}$ and the height was $4.5 \mathrm{~cm}$. The float was loaded with lead shot. The weight of the float in the air was $40.95 \mathrm{G}(0.402 \mathrm{~N})$ and in water at $23^{\circ} \mathrm{C}$ it was 5.71 $\mathrm{G}(0.056 \mathrm{~N})$. The float was suspended to a dynamometer using a fishing line with a diameter of $0.06 \mathrm{~mm}$.

The required resolution of the dynamometer is $0.01 \mathrm{G}$, which is about $0.0001 \mathrm{~N}$. The measurement range needed depends on the design of the float and is approximately 0 to $50 \mathrm{G}$. The dynamometer with hitched float is mounted on a movable arm of mechanised stand and positioned vertically with an accuracy of $1 \mathrm{~mm}$. The dynamometer measures the apparent weight of the float at intervals selected by the observer. The results are saved in the memory (maximum 5000 records). The results are also transmitted to an external recording system via USB connection.

It is important that the position of the hook of the dynamometer remains fixed during the measurement of the force. This allows the performance of measurements at

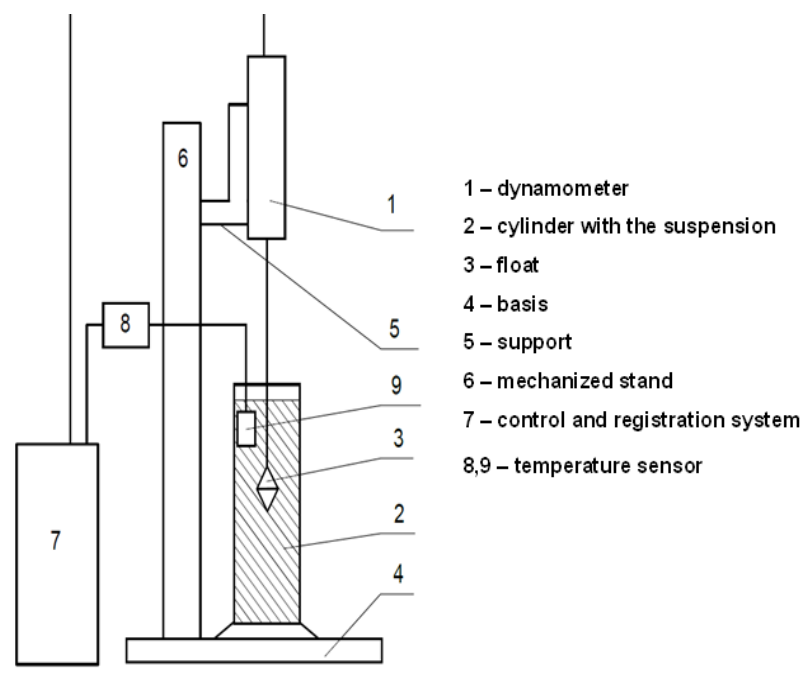

Fig. 1. Device construction scheme. 
a

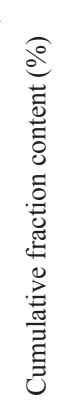

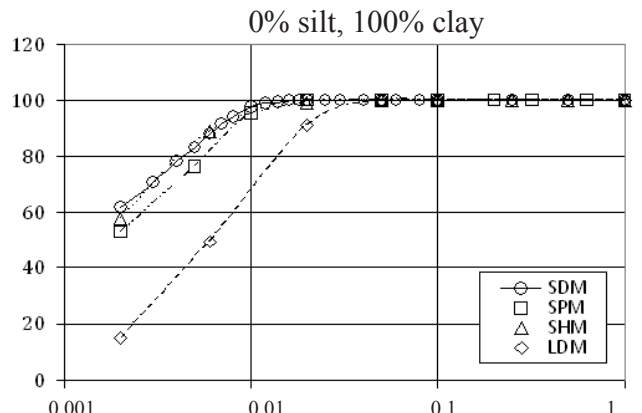

c
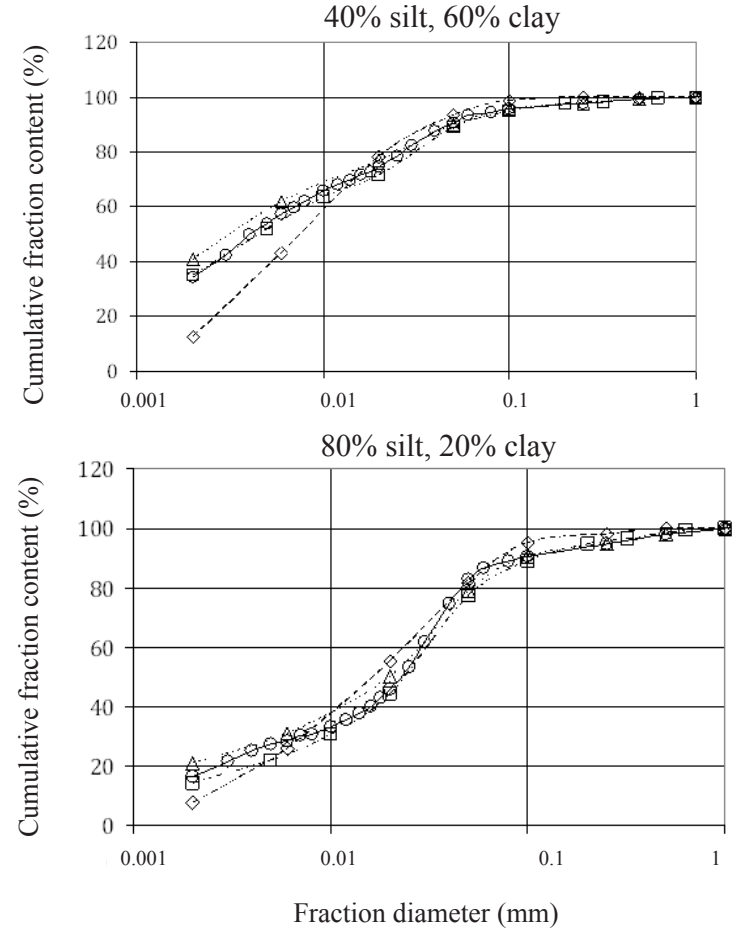

b

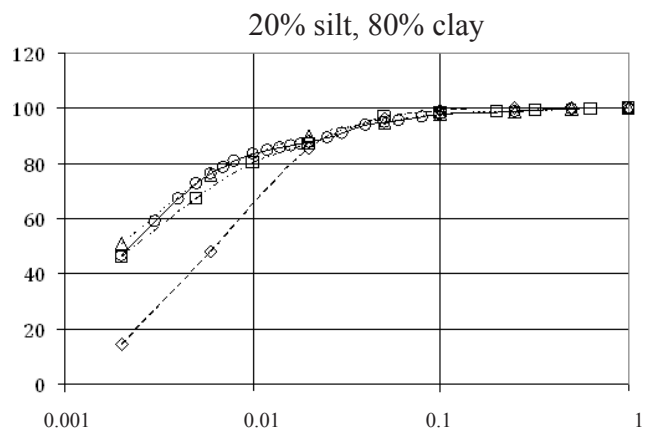

d

$60 \%$ silt, $40 \%$ clay

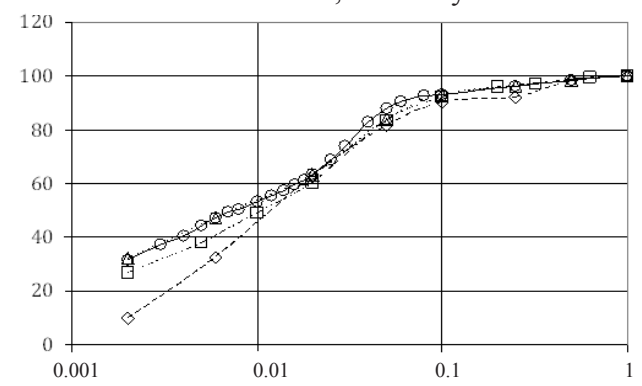

f

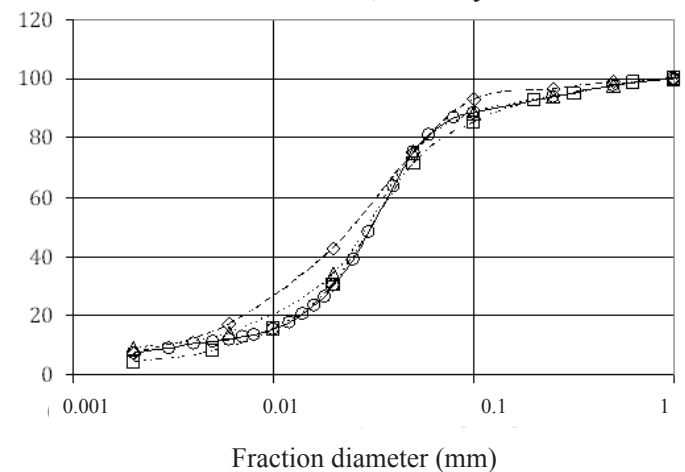

Fig. 2. Comparison of calculated and measured cumulative fraction content curves for mixtures of 'silt' and 'clay' with the predetermined proportions using SDM.

a constant depth chosen by the experimenter. The dynamometer we used was produced by the Mark10 company - model M7-012.

An external computer system controls the movement of the arm of the mechanised stand setting the float at the respective times and at the desired depths which are stated by Eq. (12). Measurement of the distance between the slide of the dynamometer and the base of the instrument is performed with an electronic ruler. Based on this distance, the depth of the centre of float buoyancy is calculated. Movement of the stand arm is carried out at two velocities. In the first phase, the arm moves at the speed of $30 \mathrm{~mm} \mathrm{~s}^{-1}$, and on reaching a depth close to the desired position the velocity is reduced in order to achieve a higher positioning accuracy.

A sensor for measuring the temperature of the soil suspension is also mounted on the movable arm of the stand. The movement is effected by a DC electric motor and a drive transmission composed of a gearwheel and bearings screw. The whole measurement system is mounted on a stable, solid base equipped with legs with a vibration damping material on the base, and a levelling system. A schematic diagram of the device construction is shown in Fig. 1.

\section{MATERIALS AND METHODS}

In order to test the proposed method of measurement the grain size composition of artificially obtained clay and silt mixtures were assessed. One of the components of these mixtures was kaolinite supplied by the Merck company, with the specific density $2.60 \mathrm{~g} \mathrm{~cm}^{-3}$ and weight loss during heating at $600^{\circ} \mathrm{C}<15 \%$. The second component was a 'silt' material from the cambic horizon of Cambisols located near the village of Stary Waliszów in the Kłodzko Valley with specific density of $2.65 \mathrm{~g} \mathrm{~cm}^{-3}$ and an organic $\mathrm{C}$ content below the detection level for the method of wet oxidation with titration by ammonium ferric sulphate. Neither component contained calcium carbonate. Further 
on, these test sample components will be conventionally named 'clay' and 'silt'. Both components were mixed in the proportions of 20 to 80,40 to 60,60 to 40 , and 80 to $20 \%$. Particle size composition of 'pure components' and mixtures were determined with the use of the sieve hydrometer method (SHM), sieve pipette method (SPM), laser diffraction method (LDM), and finally by the method described above which for the purposes of this study is defined as 'dynamometric' (SDM - sieve dynamometer method).

Prior to the analysis of the 'silt' sample, particles with diameter $>2 \mathrm{~mm}$ were dry sieved off. Fractions $<2 \mathrm{~mm}$ were used for further analysis. Due to its characteristics, the fraction $>2 \mathrm{~mm}$ was not sieved off for 'clay'. Due to the nature of the test samples, it was not necessary to remove organic matter or carbonates. Preparation of samples for analysis using the 'dynamometric' method (SDM) was carried out analogously to SHM and SPM (ISO 11277: 1998).

To increase the weight changes of the float during the measurement, samples weighing $80 \mathrm{~g}$ were used. The volume of suspension was $1000 \mathrm{~cm}^{3}$.

After preparing the test samples, measurements of the apparent weight of the float were carried out using a dynamometer with a resolution of $0.01 \mathrm{G}$ and a measurement range of $0-50 \mathrm{G}$.

Data were collected at intervals of $5 \mathrm{~s}$. The float was initially immersed to a depth of $25.5 \mathrm{~cm}$ (distance from the centre of float buoyancy to the surface of the suspension) and after $3600 \mathrm{~s}$ it was raised to a depth of $23.5 \mathrm{~cm}$, at $4000 \mathrm{~s}$ to a depth of $19 \mathrm{~cm}$, at $4300 \mathrm{~s}$ to a depth of $15 \mathrm{~cm}$, at $10300 \mathrm{~s}$ to $9 \mathrm{~cm}$ and after $15000 \mathrm{~s}$ to $5.8 \mathrm{~cm}$.

The results of measurements (Fig. 2) were smoothed in the range of $0-100 \mathrm{~s}$ using a polynomial of the second order, and for other times by fragments of linear functions. Before smoothing, the outliers from the moving averages were rejected, in order to eliminate errors resulting from vibration of measurement system. Total measurement time taken to determine the content of fractions $<0.002 \mathrm{~mm}$ was $15067 \mathrm{~s}(4 \mathrm{~h} 11 \mathrm{~m} 7 \mathrm{~s})$. The measurement data comprise 21 size fractions. The analysis was performed in 3 replications, and the results were calculated as the means of 3 measurements.

Particle size analysis using the sieve hydrometer method (SHM) was performed in 3 replications according to ISO 11277: 2005 standard, and the results are presented as averages of resultant data.

In the measurements carried out with the sieve pipette method (SPM), 12 particle size fractions were distinguished. From these fractions, 6 were measured by sieving and also 6 by pipetting. The equivalent diameters of those fractions were in the range of: $0.05-0.02,0.02-0.01,0.01$ $0.005,0.005-0.002,0.002-0.001$, and $<0.001 \mathrm{~mm}$. The reported results are the average of three replicates.

The analyses of the particle size composition by laser diffraction (LDM) were performed using a Malvern Mastersizer 2000, equipped with a Hydro MU device. The measurements were performed after mechanical homogenisation using $4 \mathrm{~min}$ of ultrasonic treatment of the suspension. Measurement was conducted for obscuration (turbidity of suspension) at a range between 10 and $20 \%$. The target value was obtained after 4 min of ultrasounds effect on the suspension. If the obscuration level exceeded the expected value, the sample was diluted by disconnecting the returning tube from the device, thus not disturbing the work of the pump, and at the same time we systematically supplied distilled water (Bieganowski et al., 2010). Measurements were conducted over a full range of particle diameters with a Malvern Mastersizer 2000 from 0.02 to $2000 \mu \mathrm{m}$. For measurements, both $\mathrm{He}-\mathrm{Ne}$ gas laser with wavelength $633 \mathrm{~nm}$ and LED light with wavelength $466 \mathrm{~nm}$ were used.

The following optical parameters were adopted: absorption index -0.1 , refractive index for solid particles -1.65 (corresponding to the value adopted for aluminium-silicates); light reflectance for water 1.33. Measurements were performed in 3 replications within $5 \mathrm{~s}$ for a single measurement, with a $3 \mathrm{~s}$ interval between the repetitions.

The algorithm for samples with irregular shape was used for calculations. For the sample of $100 \%$ 'clay' (pure kaolinite), we used the 'Multi Narrow' model and for the remaining samples the 'General Model' (Bieganowski et al., 2015; Malvern Instruction Manual, 1997).

Because of the very small doses of samples used in the measurements, special attention was paid to sample homogenisation. This was achieved by repeatedly mixing and randomising soil samples before application to the Hydro MU device.

\section{RESULTS AND DISCUSSION}

The results of measurements are presented in Fig. 2 as cumulative particle size distribution curves. Fig. $2 \mathrm{a}$ and $2 \mathrm{f}$ show the curves of $100 \%$ 'clay' and $100 \%$ 'silt' samples, while Fig. 2b, c, d, e show particle size curves for mixtures with predetermined proportions $(20: 80 ; 40: 60 ; 60: 40$ and $80: 20)$. In the case of 'clay' samples, the results achieved with the proposed dynamometric method are in close agreement with those obtained with SPM and SHM, while LDM significantly underestimates fractions of equivalent diameters - less than $0.002 \mathrm{~mm}$.

A similar underestimation of the 'clay' fraction using LDM is reported for the different soil particles by Ryżak and Bieganowski (2010) and Sochan et al. (2012) and for marine sediments and loess (Buurman et al., 2001). Underestimation of fractions with dimensions $<0.004 \mathrm{~mm}$ is also noted by Kun et al. (2013) and Beuselinck et al. (1998). Relatively close agreement of results obtained using both LD and hydrometer-sieve methods is reported by Ryżak and Bieganowski (2010). 

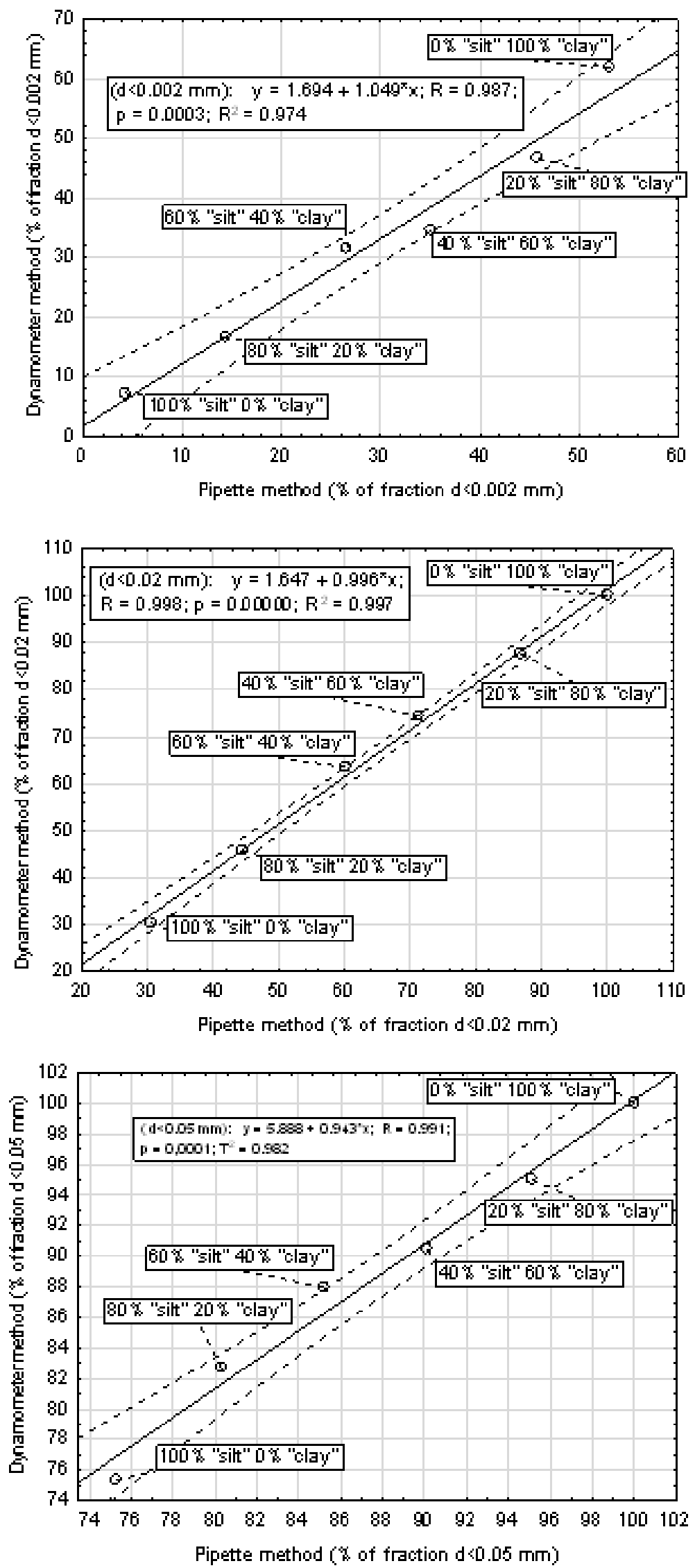

Fig. 3. Comparison of chosen fraction content measured with SPM and SDM. 

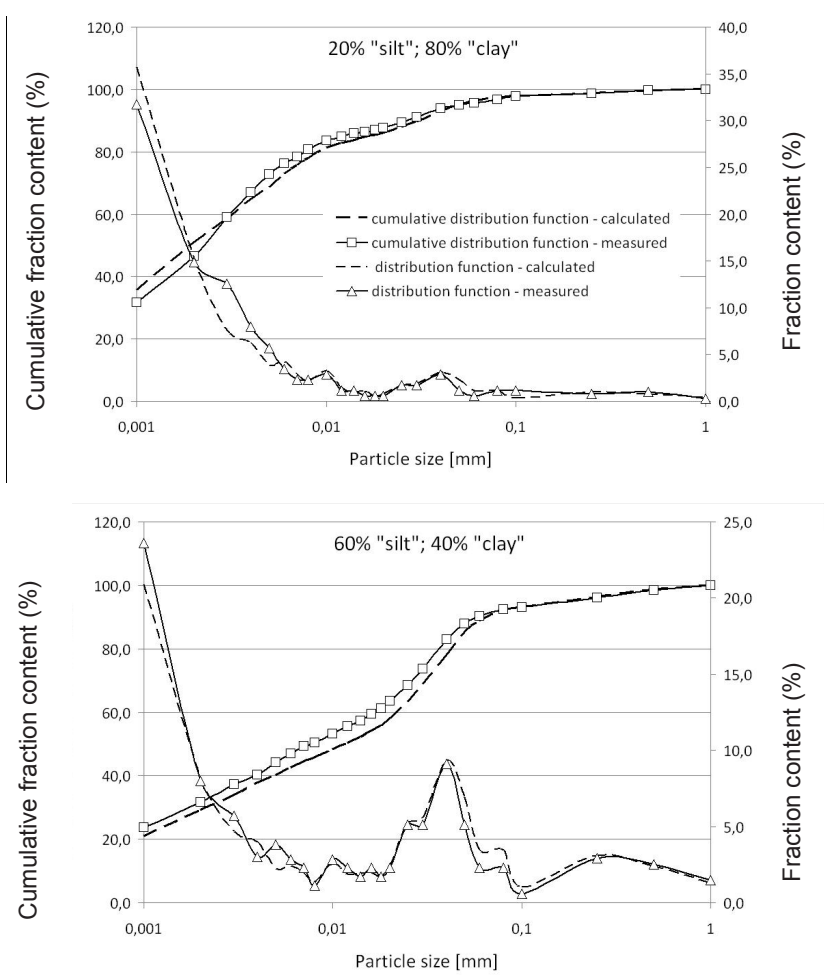
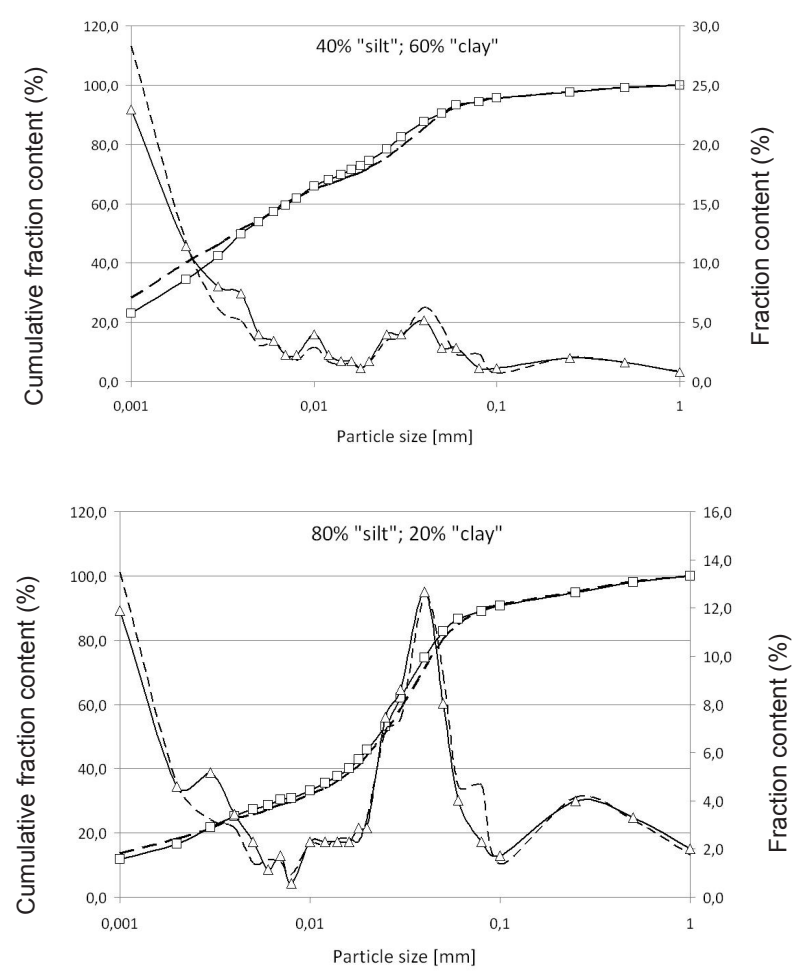

Fig. 4. Comparision of calculated and measured granulometric fraction contents for choosen soil mixtures.

Less clear results were obtained for the 'silt' sample. In this case, the described measurement method returns results which are very comparable to the other methods (Fig. 2f) wherein none of the methods compared differed from each other. Only for the fraction range between 0.006 and $0.014 \mathrm{~mm}$ did LDM give higher results than the other methods. A similar overestimation for the fraction of fine 'silt' using LDM was observed by Orzechowski et al. (2014). Additionally, Fig. 3 present a comparison of the content of fraction $<0.002$; $<0.02$ and $<0.05 \mathrm{~mm}$ measured by methods SPM and SDM. As we can see, the linear regression determination coefficients, for all examined fractions, are above 0.97 and standard error of estimation is less than $3.37 \%$.

The internal test of accuracy for each method may be the comparison of measured cumulative particle size distribution curves for mixtures of two tested samples, with the calculations being based on their known proportions in the mixture and on the particle size composition of $100 \%$ 'clay' and $100 \%$ 'silt' samples.

These results, in the form of a comparison of calculated and measured cumulative particle size distribution curves for SDM, are presented in Fig. 4.

It was observed for SDM that the maximum difference between the calculated and measured particle size distribution curves for the test samples does not exceed 5.6\% (achieved for a mixture containing 40\% 'silt' and 60\% 'clay', for particles with equivalent diameter of less than $0.002 \mathrm{~mm}$ ).

For SPM, the analogous maximum difference between the calculated and the measured content of the fractions was $4.4 \%$ (achieved for a mixture containing $20 \%$ 'silt' and $80 \%$ 'clay', for particles with equivalent diameter of less than $0.005 \mathrm{~mm}$ ). For SHM, the maximum difference between the calculated and the measured content of the fractions was $6.0 \%$ (achieved for a mixture containing $40 \%$ 'silt' and 60\% 'clay', for particles with equivalent diameter less than $0.02 \mathrm{~mm}$ ). For LDM, the maximum difference between the calculated and the measured content of fractions was $6.5 \%$ (achieved for a mixture containing $40 \%$ 'silt' and $60 \%$ 'clay', for particles with equivalent diameter less than $0.02 \mathrm{~mm}$ ).

Another accuracy test for the presented method may be the measurement of the chosen fraction carried out at different depths and at different times. Using the Eq. (13) one can calculate the measurement time for different depths for a given fraction. For example, the fraction $d<0.02 \mathrm{~mm}$ may be measured (at $20.6^{\circ} \mathrm{C}$ ) at a depth of $10 \mathrm{~cm}$ after the time of $274 \mathrm{~s}$ or at a depth of $20 \mathrm{~cm}$ after the time of $549 \mathrm{~s}$.

The measurements were performed according to the previously described methodology. Table 1 shows the statistical parameters of the measurement results of 5 fractions, for previously described clay and silt mixtures. For 
T a b l e 1. Statistical parameters of the measurement results

\begin{tabular}{|c|c|c|c|c|c|}
\hline \multirow{2}{*}{ Parameter } & \multicolumn{5}{|c|}{ Content of fraction $(\%)$ with diameters below $(\mathrm{mm})$} \\
\hline & 0.006 & 0.010 & 0.020 & 0.035 & 0.050 \\
\hline \multicolumn{6}{|c|}{$80 \%$ silt, $20 \%$ clay } \\
\hline Min-Max & $29.0-29.70$ & $32.80-35.30$ & $43.10-45.70$ & $61.20-64.20$ & $78.90-83.20$ \\
\hline Average & 29.30 & 34.50 & 44.10 & 63.10 & 81.50 \\
\hline SD & 0.28 & 0.98 & 0.96 & 1.16 & 1.67 \\
\hline CV $(\%)$ & 0.96 & 2.84 & 2.18 & 1.84 & 2.05 \\
\hline \multicolumn{6}{|c|}{$60 \%$ silt, $40 \%$ clay } \\
\hline Min-Max & $46.90-48.00$ & $51.20-53.90$ & $61.40-63.50$ & $77.40-80.10$ & $80.60-86.00$ \\
\hline Average & 47.60 & 52.80 & 62.50 & 79.00 & 83.20 \\
\hline $\mathrm{SD}$ & 0.40 & 0.96 & 0.98 & 0.96 & 1.77 \\
\hline $\mathrm{CV}(\%)$ & 0.84 & 1.82 & 1.57 & 1.22 & 2.13 \\
\hline \multicolumn{6}{|c|}{$40 \%$ silt, $60 \%$ clay } \\
\hline Min-Max & $57.70-59.80$ & $65.20-67.30$ & $72.60-74.80$ & $83.30-86.00$ & $85.50-90.80$ \\
\hline Average & 58.80 & 66.20 & 74.10 & 84.50 & 88.50 \\
\hline SD & 0.76 & 0.89 & 0.80 & 0.92 & 1.74 \\
\hline CV $(\%)$ & 1.29 & 1.34 & 1.08 & 1.09 & 1.97 \\
\hline \multicolumn{6}{|c|}{$20 \%$ silt, $80 \%$ clay } \\
\hline Min-Max & $72.50-75.10$ & $80.30-83.20$ & $88.20-91.40$ & $93.40-95.80$ & $94.20-98.70$ \\
\hline Average & 73.10 & 81.80 & 89.40 & 94.20 & 96.90 \\
\hline SD & 1.23 & 1.18 & 1.49 & 1.62 & 2.35 \\
\hline CV $(\%)$ & 1.68 & 1.44 & 1.67 & 1.72 & 2.43 \\
\hline
\end{tabular}

$\mathrm{SD}$ - standard deviation, $\mathrm{CV}$ - coefficient of variation.

each of the tested fractions, measurements were performed at depths of $6,10,15,20,25$ and $30 \mathrm{~cm}$ after properly selected times calculated from the Eq. (13).

A method operating properly should give the same results of fractions content when measured at different depths, after properly selected times resulting from Eq. (13). Ranges (max - min) presented in Table 1 do not exceed the value of $5.4 \%$. The standard deviations for the measurement of one fraction at different depths do not exceed the value of $2.35 \%$. It can be seen that a relatively large dispersion of results occurs during measurements of fractions with the largest diameter. This is related with the short measurement times for shallow immersion depths of the float (about $25 \mathrm{~s}$ for fraction $\mathrm{d}<0.05 \mathrm{~mm}$ at $6 \mathrm{~cm}$ depth of immersion) and persistent suspension turbulences.

The existence of differences may also be related to the settling of sedimenting grains on the upper surface of the float, which results in an increase in its weight for larger settling times (greater depth of measurement) and a decrease in the calculated content of the fraction. However, based on the presented data, it can be estimated that the impact of the depth measurement on result value is relatively small and for the measurement times larger than tens of seconds it is no more than $2 \%$. This is also an indication that the content of fractions with a diameter less than $0.1 \mathrm{~mm}$ should be measured at the most accessible depth to extend the time from the stir to the first reading and to reduce the impact of turbulences in the suspension. However, for fractions smaller than $0.02 \mathrm{~mm}$, it is appropriate to raise the float as close to the surface as possible to shorten the analysis time.

Obtaining correct results with the use of the presented measurement method requires the maintenance of previously indicated conditions, and additional dependencies need to be considered.

The density of water (essentially a mixture of water sodium hexametaphosphate and sodium carbonate) and the values of coefficients ' $\alpha$ ' and ' $\eta$ ' used in Eqs (9) - (14) depend on temperature. 
As a result of the conducted test calculations for the temperature range of $19-24^{\circ} \mathrm{C}$, it was found that the obtained fraction contents (size 0.002 to $0.05 \mathrm{~mm}$ ) changed by 0.4 to $0.5 \%$ with a temperature change of $1{ }^{\circ} \mathrm{C}$.

Knowledge of temperature in the course of the research is therefore important, but at the same time a slight error in its assessment (at $0.2-0.5^{\circ} \mathrm{C}$ ) does not cause significant errors in the calculation of fraction contents.

The impact of errors in measuring the weight of the float was also analysed. As previously mentioned, the resolution of the dynamometer was $0.01 \mathrm{G}$. Dynamometers with a higher resolution which would simultaneously have a capacity of at least $50 \mathrm{G}$ are not yet commercially available. For a $80 \mathrm{~g}$ sample, the change of weight of float from the state that hypothetically all soil particles are suspended in water to complete sedimentation (pure water) is $1.89 \mathrm{G}$. Thus, for $1 \%$ of the particles the change in the weight of the float is $0.019 \mathrm{G}$. Using result smoothing and interpolation, we can read the weight of the float with a resolution of the order of $0.005 \mathrm{G}$, which means that for the $80 \mathrm{~g}$ sample we are able to read the content of fractions with an accuracy of about $0.2 \%$.

Obviously, sample weight reduction results in increased error in reading the content of the fraction. For a $60 \mathrm{~g}$ sample, a float weight change of $0.014 \mathrm{G}$ occurs in a $1 \%$ fraction and it is possible to read the content of the fraction with an accuracy of about $0.3 \%$. In turn, for a $40 \mathrm{~g}$ sample, a float weight change of $0.0095 \mathrm{G}$ occurs in a $1 \%$ fraction and it is possible to read the content of the fraction with an accuracy of about $0.4 \%$.

At the same time, the authors are aware that increasing sample mass may increase the interaction between the sedimenting particles (Allen, 1997). Closer examination of this issue will require appropriate research using samples with different weights. In principle, one could improve the measurement resolution using a float of a larger volume. This would imply an increase in total float weight (a float must have a density greater than the density of water) but, as mentioned, available precision dynamometers have a maximum capacity of $50 \mathrm{G}$. Although an increase in the volume of the float is impossible without the use of a dynamometer with high resolution and a greater capacity, optimization of its shape is needed. The shortest possible measurement depth of sedimentation is equal to the distance from the tip of the float to its centre of buoyancy. In the currently used float, this distance is $58 \mathrm{~mm}$, while shape change to a helical cone should enable shortening it to about $40 \mathrm{~mm}$. This means a shortened sedimentation time for fractions $<0.002 \mathrm{~mm}$ (at $22.5^{\circ} \mathrm{C}$ ) from 15200 to $10500 \mathrm{~s}$.

The scope of application of the proposed method for 'clay' fraction is, as in all sedimentation methods, limited by the influence of Brownian motion. According to Allen et al. (1996), the lower limit of the size of measured particles is thus equal to $0.001 \mathrm{~mm}$.
At the other end of the measuring scale, ie for a grain size close to $0.1 \mathrm{~mm}$, the problem is the short time between the end of suspension stirring and the first reading. Therefore, a suitable speed of float lowering and method of data smoothing is needed.

It has been found that for a time longer than $100 \mathrm{~s}$ it is sufficient to smooth the measurement data with sections of a linear function, but for a time less than $100 \mathrm{~s}$ a smoothing polynomial of the second order is needed. The method will not encounter limitations when analysing bimodal soils, which is not evident in LDM methods (Blott and Pye, 2006). In contrast to LDM, this method does not also require any anticipatory assumptions about the shape of grain size distribution functions.

A separate problem is the impact of deviation from the spherical particle shape of soil particles on the measurement results (Eshel et al., 2004). It is possible to replace Eq. (6) with another one suitable for the shape of sedimenting particles (Dietrich, 1982); however, this requires information about their shape. A similar problem applies to all methods of grain size composition analysis (Polakowski et al., 2014).

The authors believe that the optimization of the float shape, control of suspension temperature and accurate measurement of the float immersion depth will reduce measurement errors and allow to obtain reproducible results at the level of $1 \%$.

\section{CONCLUSIONS}

Compared to the previously used sedimentation methods, the developed method has several significant advantages.

1. By measuring the density of the suspension at the desired depth chosen by researcher, the method enables shortening of the depth of sedimentation and thus significantly shortens the time of analysis - up to $3 \mathrm{~h}$.

2. The use of a dynamometer with digital recording allows the processing of the measured values of density in digital format and instant calculation of grain size composition. Possible errors of observation are, therefore, eliminated. The course of the measurement process is controlled by computer software.

3. After the start of sedimentation (mixing of suspension), measurements are performed automatically, allowing staff to use the time for other activities such as preparation of further samples. Proper management of time and changing cylinders with suspension allows measurements with one device in several cylinders simultaneously.

4. As indicated by performed test measurements, the results obtained with the proposed method are reproducible and exhibit close agreement with those obtained with the use of the pipette method which is regarded as the reference method. In comparison to laser diffraction methods, 
the proposed solution is cheaper, and the results obtained are more closely compatible with other sedimentation methods.

5. The physical phenomenon used is the same as that which defines the character and properties of naturally occurring sediments.

Conflict of interest: The Authors declare no conflict of interest.

\section{REFERENCES}

Agrawal Y.C., McCave I.N., and Riley J.B., 1991. Laser diffraction size analysis. In: Principles, Methods and Applications of Particle Size Analysis (Ed. J.P.M. Syvitski). Cambridge University Press, UK.

Allen T., 1997. Particle size measurement. Vol. 1, Chapman and Hall, London, UK.

Allen T., Davies R., and Scarlett B., 1996. Particle size measurement. Vol. 2, Chapman and Hall, London, UK.

Bah A.R., Kravchuk O., and Kirchhof G., 2009. Fitting performance of particle-size distribution models on data derived by conventional and laser diffraction techniques. Soil Sci. Soc. America J., 73, 1101-1107.

Beusenlick L., Govers G., Poesen J., Degraer G., and Froyen L., 1998. Grain size analysis by laser diffractometry: comparison with sieve-pipette method. Catena, 32, 193-208.

Bieganowski A., Malý S., Frąc M., Tuf I.H., Váňa M., Brzezińska M., Siebielec G., Lipiec J., and Šarapatka B., 2015. Particle size distribution measured by laser diffraction method. In: Laboratory manual. Central Institute for Supervising and Testing in Agriculture, Brno, Czech Republic.

Bieganowski A., Ryżak M., and Witkowska-Walczak B., 2010. Determination of soil aggregate disintegration dynamics using laser diffraction. Clay Minerals, 45, 23-34.

Bieganowski A. and Walczak R.T., 2004. Specificity of agrophysical measurements. Problem of standardization and validation of methods. In: Basic problems of agrophysics (Eds D. Matyka-Sarzyńska, R,T. Walczak). Institute of Agrophysics PAS, Lublin, Poland.

Blott S.J. and Pye K., 2006. Particle size distribution analysis of sand-sized particles by laser diffraction: an experimental investigation of instrument sensitivity and the effects of particle shape. Sedimentology, 53(3), 671-685.

Buurman P., Pape Th., Reijneveld J.A., De Jong F., and Van Gelder E., 2001. Laser-diffraction and pipette-method grain sizing of Dutch sediments: correlations for fine fractions of marine, fluvial, and loess samples. Netherlands J. Geosciences, 80, 49-57.

Di Stefano C., Ferro V., and Mirabile S., 2010. Comparison between grain-size analyses using laser diffraction and sedimentation methods. Biosys. Eng., 106, 205-215.

Dietrich W., 1982. Settling velocity of natural particles. Water Res. Res., 18(6), 1615-1626.

Eshel G., Levy G.J., Mingelgrin U., and Singer M.J., 2004. Critical evaluation of the use of laser diffraction for particle-size distribution analysis. Soil Sci. Soc. Am. J., 68, 736-743.
Gee G.W. and Bauder J.W., 1979. Particle-size analysis by hydrometer: A simplified method for routine textural analysis and a sensitivity test of measurement parameters. Soil Sci. Soc. Am. J., 43, 1004-1007.

Gliński J. and Konstankiewicz K., 1991. Methods and apparatus for agrophysical research (in Polish). Problemy Agrofizyki 64, Ossolineum Wrocław, Polska.

ISO 11277:1998. Soil quality - Determination of particle size distribution in mineral soil material - Method by sieving and sedimentation.

Komornicki T. and Jakubiec J., 1978. Remarks on the areometric method for soil mechanical analysis as modified by $\mathrm{M}$. Prószyński. Part 5: A comparison of several granulometric methods. Acta Agraria et Silvestria. Series Agraria, 1, 83-95.

Konert M. and Vandenberghe J., 1997. Comparison of laser grain size analysis with pipette and sieve analysis: a solution for the underestimation of the clay fraction. Sedimentology, 44, 523-535.

Kovács B., Czinkota I., Tolner L., and Czinkota G., 2004. The determination of particle size distribution (PSD) of clayey and silty formations using the hydrostatic method. Acta mineralogica-petrographica, 45, 29-34.

Kovács B., Czinkota I., Tolner L., and Czinkota G., 2006. Fit method for calculating soil particle size distribution from particle density and settling time data. Agrokémia és Talajtan, 55. 295-304.

Kun A., Katona O., Sipos G., and Barta K., 2013. Comparison of pipette and laser diffraction methods in determining the granulometric content of fluvial sediment samples. J. Environ. Geography, 6, 49-54.

Loth E., 2008. Drag of non-spherical solid particles of regular and irregular shape. Powder Technology, 182(3), 342-353.

Malverm Mastersizer 2000 Instruction Manual, 1997.

Nemes A., Czinkota I., Czinkota G., Tolner L., and Kovács B., 2002. Outline of an automated system for quasy-continous measurement of particle size distribution. Agrokémia és Talajtan, 51, 37-46.

Oden S., 1915. Eine neue Methode zur mechanischen Bodenanalyse. Int. Mitt. Bodenanal., 5, 257-311.

Oliveira J.M.C., Vaz C.M.P., Reichardt K., and Swartzendruber D., 1997. Improved soil particle-size analysis by gamma-ray attenuation. Soil Sci. Soc. Am. J., 61, 23-26.

Orzechowski M., Smólczyński S., Długosz J., and Poźniak P., 2014. Measurements of texture of soils formed from glaciolimnic sediments by areometric method, pipette method and laser diffraction method. Soil Sci. Annual, 65(2), 72-79.

Polakowski C., Sochan A., Bieganowski A., Ryżak M., Földényi R., and Tóth J., 2014. Influence of the sand particle shape on particle size distribution measured by laser diffraction method. Int. Agrophys., 28, 195-200.

Ryżak M., Bartmiński P., and Bieganowski A., 2009. Methods for determination of particle size distribution of mineral soils (in Polish). Acta Agrophysica, 175, 1-97.

Ryżak M. and Bieganowski A., 2010. Determination of particle size distribution of soil using laser diffraction - comparison with areometric method. Int. Agrophys., 24(2), 177-181. 
Rząsa S., 1978. A sedimentative apparatus for a total analysis of the mechanical composition of the soil. Advances Agric. Sci. Problem Issues, 220(2), 513-524.

Rząsa S. and Owczarzak W., 2013. Methods for the granulometric analysis of soil for science and practice. Polish J. Soil Sci., 46(1), 1-50.

Singer J.K., Anderson J.B., Ledbetter M.T., McCave I.N., Jones K.P.N., and Wright R., 1988. An assessment of analytical techniques for the size analysis of fine-grained sediments. J. Sediment. Petrol., 58, 534-543.
Sławiński C., Witkowska-Walczak B., Lipiec J., and Nosalewicz A., 2011. Effect of aggregate size on water movement in soils. Int. Agrophysics, 25, 53-58.

Smith K.A. and Mullins C.E., 1991. Soil analysis. Physical Methods. Marcel Dekker, New York, USA.

Sochan A., Bieganowski A., Ryżak M., Dobrowolski R., and Bartmiński P., 2012. Comparison of soil texture determined by two dispersion units of Mastersizer 2000. Int. Agrophys., 26, 99-103. 